\title{
Some morphological aspects of myocardial bridges
}

\author{
${ }^{*} 1,2$ Mihail Tasnic, ${ }^{2}$ Ilia Catereniuc \\ ${ }^{1}$ Department of Cardiology and Interventional Cardiology, International Hospital Medpark \\ ${ }^{2}$ Department of Anatomy and Clinical Anatomy, Nicolae Testemitanu State University of Medicine and Pharmacy \\ Chisinau, the Republic of Moldova
}

\author{
Authors' ORCID iDs, academic degrees and contribution are available at the end of the article \\ ${ }^{*}$ Corresponding author: mihaitasnic@gmail.com \\ Manuscript received February 02, 2021; revised manuscript April 12, 2021; published online April 28, 2021
}

\begin{abstract}
Background: Myocardial bridges are variants of the intramyocardial position of the coronary arteries. In the specialty literature, hot topics in cardiovascular field are myocardial infarction and non-obstructive coronary artery disease with frequent connection with myocardial bridges.

Material and methods: The morphological study was based on the analysis of 200 human hearts and fragments of coronary arteries. The retrospective study was focused on the analysis of 6168 coronary angiography reports, to identify patients with myocardial bridges, their preferred location, the degree of systolic stenosis, the association between myocardial bridges and proximal to bridge and under the bridge coronary atherosclerosis.

Results: The complete myocardial bridges were described in $62 \%$ of the analyzed hearts and only in $5.3 \%$ of the total number of studied coronarographies. In the majority of cases, the complete myocardial bridges covered the anterior interventricular branch. The degree of subpontine arterial systolic stenosis varied within 10-95\%. The comparative study did not determine any correlations between the degree of subpontine vascular compression and the degree of the left ventricular myocardial hypertrophy. In $32 \%$ of cases were described proximal to bridge atherosclerotic plaques and only in one case (0.5\%) distal to bridge atherosclerotic plaques, located immediately under the bridge.

Conclusions: The research findings underline the differences in anatomical and angiographic incidence of myocardial bridges, and the inability of all bridges to reduce the lumen of under bridged artery. Current study emphasizes attention to the topography of bridges, the correlation with ventricular hypertrophy and coronary atherosclerosis.

Key words: myocardial bridge, myocardial ischemia, myocardial hypertrophy, coronary atherosclerosis.
\end{abstract}

\section{Cite this article}

Tasnic M, Catereniuc I. Some morphological aspects of myocardial bridges. Mold Med J. 2021;64(2):58-64. https://doi.org/10.52418/moldovanmed-j.64-2.21.11.

\section{Introduction}

Heart pathology takes the leading place among the diseases, with the highest rate of morbidity and mortality worldwide.

The certain anomalies and anatomical variants of the heart arteries in a certain circumstance, can cause acute and chronic coronary events, myocardial ischemia during physical effort or after it [1]. Myocardial bridges are variants of the intramyocardial position of the coronary arteries [2].

In the specialty literature, it currently becomes a forgotten cause of hot topics in cardiovascular field like myocardial infarction and non-obstructive coronary artery disease (MINOCA) [3] with frequent connection between myocardial bridges and other acute cardiac events, such as: chest pain, malignant arrhythmias, complete atrioventricular block, sudden cardiac death [4-7].

In muscular bridges there is an temporary coronary luminal narrowing. If a patient has a endothelial injury, acute myocardial infarction may occur. Smoking history and nicotine could have damaged the endothelial structure in the bridged segment [5] .
The incidence of myocardial bridges, in the analyzed hearts, as result of necropsies, reaches up to $80-86 \%[4,6]$. Not all myocardial bridges are able to cause heart ischemia, so the bridges that were determined by angiography are detected only in $0.5-33 \%$ of all analyzed coronary angiographies [5], and 3.5-38.5\% in coronary CT angiocardiographies $[6,8]$.

The mechanisms of the myocardial bridges' occurrence are not yet clear, as well as the morphological and functional features of the bridges, which can affect coronary circulation.

The need for an in-depth morphological and clinical study of the problem of morphoclinical correlations of large coronary arteries and their intramural trajectory is dictated by the widespread use of numerous procedures for assessing the subepicardial coronary vessels by minimally invasive methods.

The aim of the current study is to investigate the morphological macroscopic and angiographic aspects of myocardial bridges. 


\section{Material and methods}

The prospective-morphological study was based on the analysis of 200 human hearts and fragments of coronary arteries applying the thin anatomical dissection and morphometry. The morphological and topographic aspects of the myocardial bridges were analyzed. The retrospective study was focused on the analysis of 6168 coronary angiography reports, performed in patients suspected with coronary ischemic pathology, in order to identify patients with myocardial bridges with and without association with atherosclerotic heart pathology.

The current morphological study investigated the anatomical and angiographic incidence of the variants of the intramural trajectory, the location, the dimensions of the myocardial bridges, the association of the variants of the intramural trajectory of the heart arteries.

In the retrospective study of coronary angiography of patients with myocardial bridges there were evaluated: the preferred location of the myocardial bridges, the degree of systolic stenosis, the association between myocardial bridges and proximal to bridge and under-bridged coronary atherosclerosis. The obtained data were statistically processed.

\section{Results and discussion}

The complete myocardial bridge was defined when a portion of the subepicardial coronary artery, on one or more portions of its path, enters the myocardium with its subsequent reappearance, under the epicardium.

The complete myocardial bridges were described in $62 \%$ of the analyzed hearts.

In morphological aspect myocardial bridges were classified according the following criteria: width (wide/narrow);

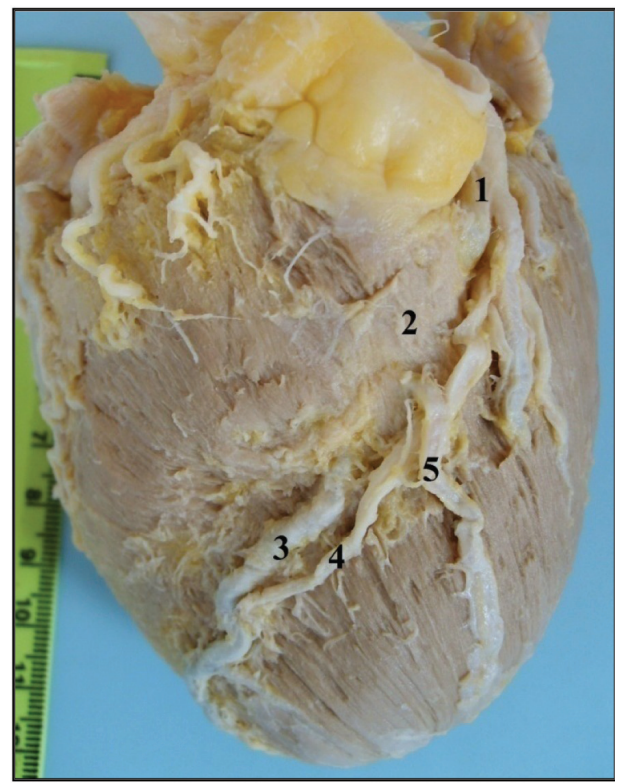

Fig. 1. Myocardial bridge covering half of anterior interventricular branch

1 - proximal to bridge arterial segment; 2 - complete myocardial bridge; 3 - distal to bridge arterial segment;

4 - vena cordis magna; 5 - second diagonal branch. thickness (thin/thick); the vessel involved (arterial/venous / arteriovenous); origin of muscle bundles (atrial/ventricular); histoarchitectonics of the myocardial bridge (muscle/ muscular and connective).

The thickness of the complete myocardial bridges was $2-5 \mathrm{~mm}-62 \%$, in $27 \%$ of cases the thickness of the bridges was up to $2 \mathrm{~mm}$. Myocardial bridges with a thickness of 6-9 $\mathrm{mm}$ are described in $15 \%$ of cases, and very thick myocardial bridges with a thickness of more than $10 \mathrm{~mm}$ - in 3\% of cases. The thick myocardial bridges, up to $1 \mathrm{~cm}$, were found exclusively on the trajectory of the middle third of the anterior interventricular branch (fig. 1). Some isolated cases of localization of the intramural segment of the artery have been determined in the immediate vicinity of the left ventricular cavity (fig. 2).

Complete myocardial bridges, usually related to the ventricular muscles, only occasionally were determined as formations similar to the origin and insertion of muscle bundles in the atrial myocardium.

In the majority of cases the complete myocardial bridges cover the anterior interventricular branch, succeeded by the diagonal branches, first marginal branch and the posterior interventricular branch. In only one case the complete myocardial bridge was detected on the path of the main trunk of the right coronary artery and its branches. On the trajectory of the left circumflex branch were found only muscle loops with an atrial or marginal ventricular origin and insertion, while along the atrial vessels complete myocardial bridges were not identified (fig. 4).

The performed study shows that, most commonly complete myocardial bridges cover the distal portion of the proximal third and also the proximal and middle portions of the middle third of the anterior interventricular branch

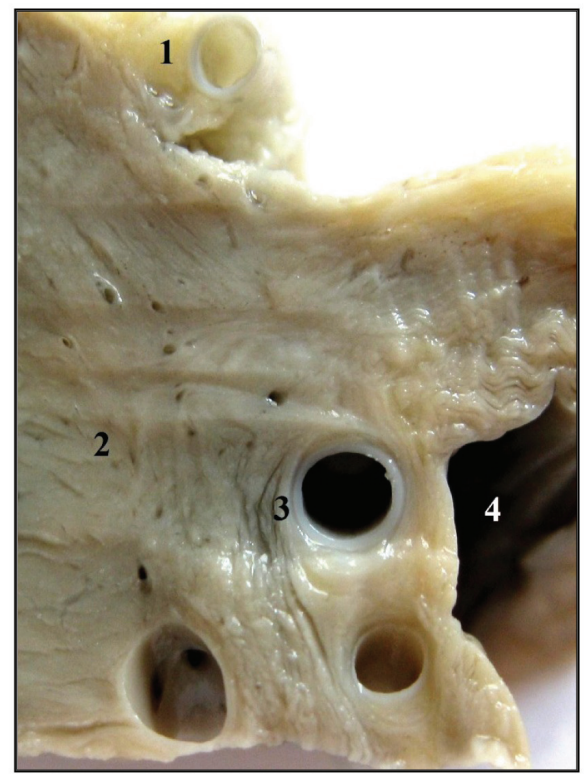

Fig. 2. Cross-section through the thick myocardial bridge covering middle portion of the anterior interventricular branch 1 - first diagonal branch; 2 - septal myocardium;

3 - under the bridged arterial part; 4 - the cavity of left ventricle. 
( $86 \%$ of cases with complete myocardial bridge on the anterior interventricular branch). Rarely, in 32\% and $26 \%$ of cases complete myocardial bridge covers the distal and proximal third of the LAD as well.

The widest (up to $70 \mathrm{~mm}$ ) and thickest (up to $8-10 \mathrm{~mm}$ ) of complete myocardial bridges were detected along the anterior interventricular branch. Only in $4 \%$ of cases were found subtotal complete myocardial bridges, covering the anterior interventricular branch over a distance exceeding $75 \%$ of the total vessel length.

In $75 \%$ of the studied hearts, several complete myocardial bridges were located in the same heart.

The most frequent association is between the complete myocardial bridge on the trajectory of the anterior interventricular branch and the complete myocardial bridges located on the first marginal branch. Such associations were detected in $21 \%$ of the investigated cases. Two myocardial bridges were detected in $33 \%$ of cases. In $18 \%$ of cases 3 myocardial bridges were located in the same heart, and in $3 \%$ of cases 4 and more bridges were determined in one heart. The maximum number of the complete myocardial bridges detected per one organ is 7 .

The analysis of 6168 reports of diagnostic coronary angiography performed on people with suspected severe coronary atherosclerotic pathology, myocardial bridges were detected in 331 of people, constituting $5.3 \%$ of the total number of cases.

A significant discrepancy between the morphological and angiographic frequency of screening for the myocardial bridge is explained only by the inability of all myocardial bridges to reduce the lumen of the artery during systole for various reasons.

Based on the study, it was found that the number of myocardial bridges reported by interventional cardiologists during this period varied from year to year, due to a lack of information. In many cases, the mention of myocardial bridges detected in coronary angiography was considered by some specialists as harmless anatomical variants, which do not require to be mentioned in medical documentation.

The study group was underestimated in patients with myocardial bridges and coronary arteries without severe coronary lesions and in patients with severe coronary lesions. In this way, in the category of patients without moderate and severe coronary artery disease, myocardial bridges were detected in $56 \%$ of men and $43 \%$ of women, unlike the group of patients with severe coronary heart disease - in $82 \%$ of cases patients with severe coronary atherosclerosis and myocardial bridges are men and only $17 \%$ - women. This correlation is within the natural ratio of the distribution of atherosclerotic heart diseases by gender, therefore there is no significant difference in the prevalence of myocardial bridges by gender.

The angiographic identification of myocardial bridges during angiographies is possible only by the direct pontine effect on the underlying vessel - systolic compression, in other words - the squeezing effect, "milking" of the blood column under the bridge (fig. 3).
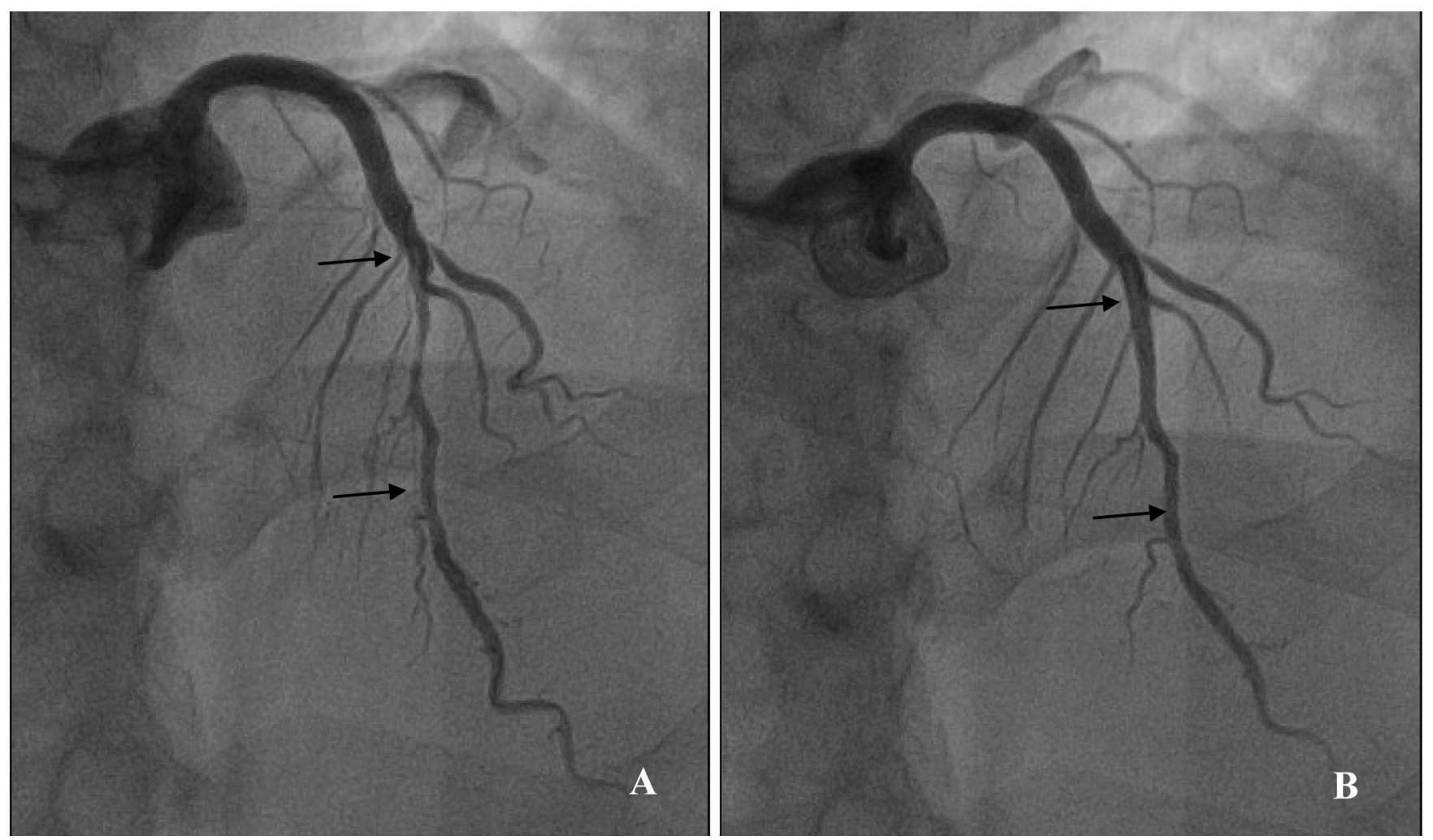

Fig. 3. The compression of the middle portion of anterior interventricular branch caused by myocardial bridge. Images from coronarography

A - during systole of the heart, B - during diastole of the heart 
The degree of systolic stenosis is directly proportional to the compression force of the pontine myocardium and inversely proportional to the resistance of the vascular wall, the diameter of the vessel lumen, and the intracoronary pressure.

In the case of bridges that cause the reduction of the vascular lumen up to $50 \%$, the intramural portion of the vessel, at the time of maximum systole, was homogeneously stenotic, having uniform vascular contours. In the case of subtotal systolic compression, the subpontine vascular segment had the appearance of a "sawfish", with the alternation of narrow vascular portions and wider ones.

The non-uniformity of the systolic stenosis of the artery can be caused by the arrangement of the pontine myocardial bundles under the different angles and / or the variation of the areas of anti-systolic resistance of the vascular wall and the tissue structures in the subpontine perivascular space.

Arterial stenosis caused by myocardial bridge is not always limited to systole, but also persists in diastole; can be a part of the diastole or it can be permanent.

In the longitudinal section plane through the muscleartery complex, the angiographic view in maximal systole takes shape of a "trough".

Often, during the routine coronary examination, the middle and distal portion of the LAD do not show obvious systolic stenoses, but have a "trough" like deformity, which would correspond to the vascular deformation caused by the involvement of the artery under the myocardial bridge, but which, in this case, is systolic inactive. In such cases, it may be appropriate to perform tests to induce coronary spasm (with acetylcholine) and to reduce intracoronary pressure (with nitroglycerin) to detect the activity of myocardial bridges, or to demonstrate the possibility of inducing the vascular spasm in the subpontine portion.

Out of the 331 cases of patients detected with myocardial bridges, in $97 \%$ of cases they were located along anterior interventricular branch, and in $3.6 \%$ of cases - on other vessels: right coronary artery, circumflex artery, first diagonal branch, marginal branches, posterolateral branch (fig. 4).

Bridges located in the same plane on anterior interventricular branch and diagonal branches were described, being covered by the same "myocardial flap". Similar bridges were found on the trajectory of diagonal branch, intermediate branch and marginal branch, with a shape of myocardial strips located in the same plane, like if they were covered by the same "myocardial flap".

In $65 \%$ of cases, the bridges were located in the middle third of the anterior interventricular branch, and in $27 \%$ the myocardial bridges were covering the distal third of the artery. In $4.23 \%$ of cases, the bridges located on the LAD were detected as extended, covering 2 segments at the same time.

The degree of subpontine arterial systolic stenosis varies within $10-95 \%$. From the total number of described myocardial bridges, in $50 \%$ of cases they were causing insignificant systolic compression of the artery, reducing the lumen of the vessel up to $50 \%$ of the initial value (visually appreci-
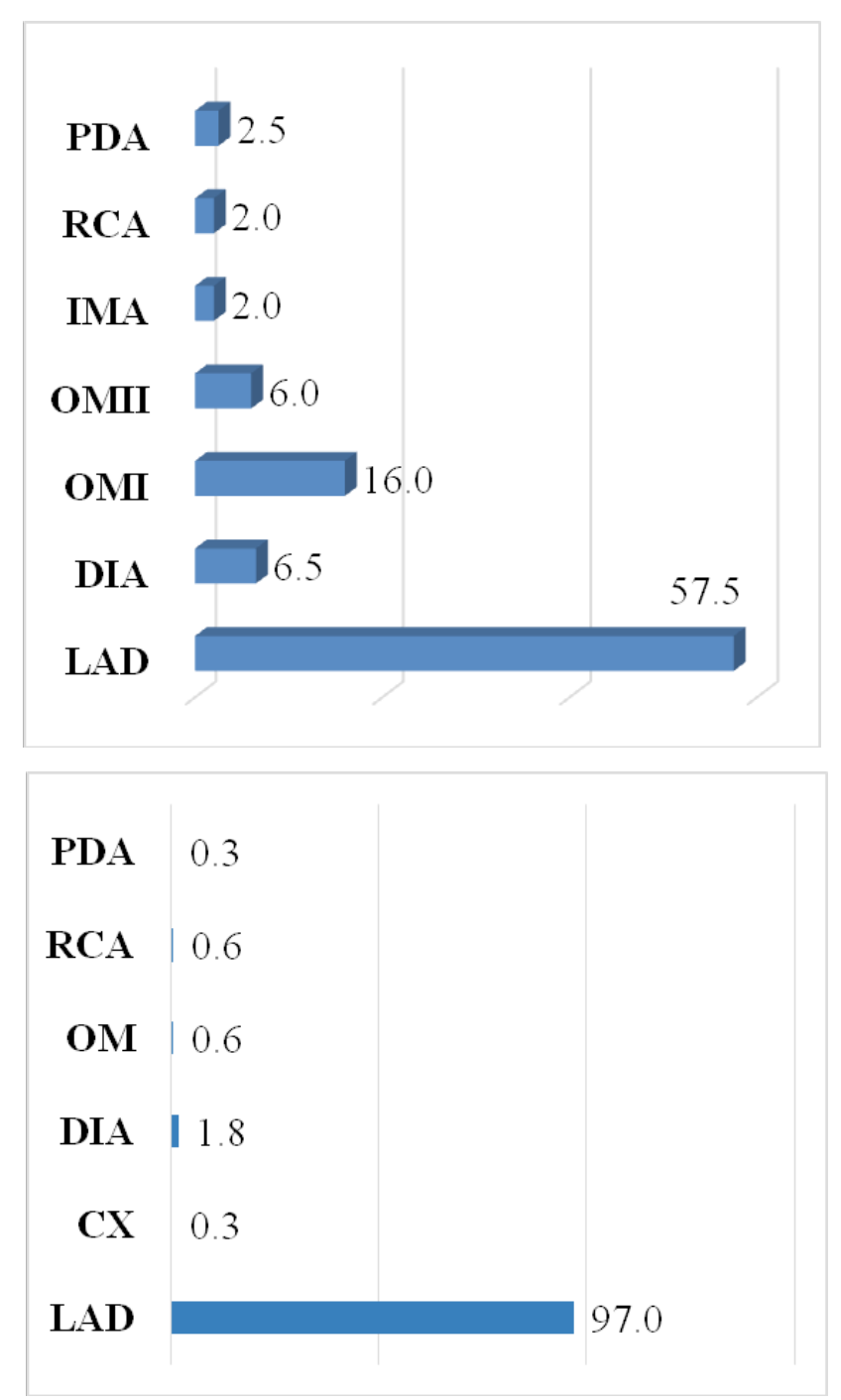

Fig. 4. The topography of myocardial bridges discovered during morphological examination of human hearts and anatomical pieces of coronary arteries (the diagram above), myocardial bridges discovered during coronarographies (the diagram below) PDA - posterodiagonal branch (posterior interventricular branch); RCA- right coronary artery; OM - obtuse marginal branch; DIA - diagonal branch; CX - circumflex artery; $\mathrm{LAD}$-anterior descendent branch.

ated) and only in $16 \%$ of cases the degree of compression exceeded $75 \%$.

Another widely discussed aspect in the literature was analyzed as well - the interdependence of the degree of systolic stenosis of the anterior interventricular branch and the degree of myocardial bridge hypertrophy. The comparative study did not determine any correlation between the degree of subpontine vascular compression and the degree of left ventricular myocardial hypertrophy in the main study group.

Consecutively, has also been investigated the correlation between myocardial bridges and proximal to bridge coronary atherosclerotic lesions. From the total examined reports, in $32 \%$ of cases were described proximal to bridge 
atherosclerotic plaques located at various distances proximal to the myocardial bridge; and only in one case $(0.5 \%)$ - distal to bridge; atherosclerotic plaques located immediately under the bridge. Coronary atherosclerosis was not detected in intramural part of the vessel.

The correlation between the degree of dynamic subpontine coronary stenosis and the degree of proximal to bridge atherosclerosis was also not determined.

\section{Discussion}

The morphological and clinical study is based on statistically significant number of the research objects, which made possible to formulate statistically true conclusions for the morphological and functional aspects of myocardial bridges.

The obtained morphological data is related with the data reported in multiple studies dedicated to myocardial bridges and the particular location of bridges on the trajectory of the anterior interventricular branch $[8,9]$.

The discrepancies between the anatomical and angiographic (clinical) incidence are dictated by the inability of the bridges to compress the arteries during the systole. The ability of the myocardial bridge to reduce the lumen of the artery during cardiac contraction is determined by the thickness of the myocardial bridge, width, histological composition, the caliber of the vessel covered by the bridge, the orientation of the myocardium around the bridge $[10,11]$. All the mentioned features were the basis for the elaboration of the own morphological classification of the myocardial bridges.

The detection of the myocardial bridges in all branches of the coronary arteries shows that there are no arteries predisposed to intramural localization but their formation is determined in ontogenesis and would correspond to incomplete myocardial resorption around the arteries of the heart [6].

A special attention gets the fact of the association of myocardial bridges on the same vessel or in the same heart, which is detected in $75 \%$ of cases by the possibility of amplifying the proischemic effect $[9,10]$.

Although, in most cases, were described thin myocardial bridges, in the rare cases - 3\%, were found thick myocardial bridges of about $10 \mathrm{~mm}$, with a deep localization of the anterior interventricular branch, in the thickness of the interventricular septum. The incidence coincides with the angiographic incidence of myocardial bridges within 5\%, suggesting that only thick bridges could reduce the lumen of the vessel.

An angiographic incidence of $5 \%$ versus $62 \%$ of morphological one suggests that thick myocardial bridges can be considered as coronary arteries anomalies, as they cause visible narrowing on angiograms of the intramural coronary segment. In the literature, the term "anomaly" is often referred to myocardial bridges [10], referring them to the group of structures with negative effects.

The angiographic view of the myocardial bridges coincided with those described in the literature. Also, within this particular study a special attention was given to the nonuniformity of compression of the coronary arteries during the systole, which is suggesting the non-uniformity of the pontine compression forces determined by the orientation of the pontine myocardium, the thickness of the bridge and possibly by the pontine histoarchitectonics, and the correlation between the amount of the connective and muscular tissue.In $50 \%$ of detected cases the degree of subpontine systolic coronary reduction was below $50 \%$ and only in $16 \%$ of cases was over $75 \%$.

However, not all patients with severe subpontine stenosis caused by myocardial bridges develop myocardial ischemia, possibly some other proischemic mechanisms, are intervening, such as subpontine coronary spasm which is so intensely attributed to the intramural trajectory by some authors [10-12]. To the genesis of subpontine coronary spasm often are attributed: stress, smoking, drug use $[13,14]$ and chemotherapy treatment [15]. All above-mentioned risk factors are linked with toxic injury of functional integrity of the heart. Understanding the mechanisms underlying cardiotoxicity may lead to treatment of the toxicity or to its prevention. There is a field for harm reduction strategies to be applied by reducing or eliminating effects of acute cardiotoxicants on the body.

$20 \%$ of deaths attributed to coronary diseases are linked to smoking [16]. Toxic effects of smoking result from chronic exposure to numerous toxic chemicals and carcinogens, including cardiotoxicants in tobacco smoke following the combustion of tobacco in the cigarette. Conversely, products that do not involve combustion to deliver nicotine (e.g. nicotine replacement therapies (NRTs), smokeless tobacco, or electronic cigarettes) have substantially reduced levels of toxic substances [17]. In smokeless heated tobacco products (HTPs) levels of carbon monoxide, acute heart toxicant, is reduced up to $98 \%$ compared to cigarettes, as well as aldehydes (approximately by $80-95 \%$ ) and VOCs (approximately by 97-99\%) [18]. Non-combustible nicotine sources are considered for smoking harm reduction [19]. Giuseppe Biondi-Zoccai et al. made independent assessment of proatherosclerotic effects of cigarette smoking versus HTP use [20]. HTP had less impact than conventional cigarette on some dimensions of oxidative stress, antioxidant reserve, platelet function, and blood pressure. In addition, HTP had less acute effects on soluble Nox2-derived peptide, 8-isoPGF2a-III (quantification of isoprostanes) and vitamin E. Also, users of HTP appeared to be more satisfied and capable of decreasing desire for continuing smoking.

Promoting smoking cessation should become an essential contributor to the treatment of patients with CVD and especially patients with coronary atherosclerosis, myocardial bridges founded after coronary angiography. In cases when patients do not quit smoking, doctors should apply harm reduction strategies, that is, to discuss with a patient the possibility of switching to an alternative products with a reduced or modified risk, while continuing efforts to quit the use of nicotine completely, since alternative products reduce, but do not entirely eliminate adverse health effects [21]. 
The study showed the presence of proximal to bridge atherosclerotic plaques in $32 \%$ of cases, which corresponded to data from literature, but there wasn't any correlation between the degree of pontine stenosis and the atherosclerotic stenosis degree. Thus, even if the myocardial bridges predispose to the formation of proximal to bridge coronary atherosclerosis by generating the retrograde blood flow with a traumatic mechanical effects for the intima in the proximal to bridge portion of coronary artery being a predisposing factor for coronary atherosclerotic pathology (depending on the thickness and length of the bridge) $[8$, 22], these structures are not able to determine the grade of coronary lesion and the grade of prepontine atherosclerotic stenosis.

At the same time, no cases of subpontine atherosclerosis were detected, all the obtained data being consistent with the information given in the literature and is suggesting the protective effect of the bridges in under the bridge coronary atherogenesis [11, 12, 23].

There was not determined any correlation between the grade of the ventricular myocardial hypertrophy and the degree of systolic compression caused by the bridges.

Although, many authors stipulate that myocardial hypertrophy predisposes to thickening of the bridge and increases the incidence of their detection on the coronary angiography, especially in the case of hypertrophic cardiomyopathy, according to obtained results only the severe hypertrophy characteristic for the hypertrophic cardiomyopathy, would increase the capabilities of the myocardial bridge to compress the artery into cardiac systole and not a hypertrophy of up to $20 \mathrm{~mm}$ [24].

\section{Conclusions}

The anatomical incidence of myocardial bridges is $62 \%$, and the angiographic one $-5.3 \%$ in the analyzed cases. The discrepancy in the incidence is determined by the myocardial bridges' capacity to reduce the under-bridged arterial segment lumen.

The coronary branch with the highest predisposition to myocardial bridges is the anterior interventricular branch. This vessel was the location of the thickest bridges detected in this research.

The stenosis' degree caused by myocardial bridges varies depending on its compression force between $10-95 \%$. The most compressive bridges were detected on the anterior interventricular branch trajectory.

No correlation was found between the degree of systolic stenosis caused by the myocardial bridges and the prepontine atherosclerosis degree. Similarly, no associations were found with the degree of the left ventricular myocardium hypertrophy.

\section{References}

1. Jiang X, Zhou P, Wen C, Yin Z, Liu T, Xu M, et al. Coronary anomalies in 11267 Southwest Chinese patients determined by angiography. BioMed Res Int. 2021;2021:1-7. doi: 10.1155/2021/6693784.
2. Teragawa $\mathrm{H}$, Oshita $\mathrm{C}$, Ueda T. The myocardial bridge: potential influences on the coronary artery vasculature. Clin Med Insights Cardiol. 2019;13. doi: 10.1177/1179546819846493.

3. Okada K, Hibi K, Ogino Y, Maejima N, Kikuchi S, Kirigaya H, et al. Impact of myocardial bridge on life-threatening ventricular arrhythmia in patients with implantable cardioverter defibrillator. J Am Heart Assoc. 2020;9(21):e017455. doi: 10.1161/JAHA.120.017455.

4. Matta A, Canitrot R, Nader V, Blanco S, Campelo-Parada F, Bouisset F, et al. Left anterior descending myocardial bridge: Angiographic prevalence and its association to atherosclerosis. Indian Heart J. 2021. https://doi. org/10.1016/j.ihj.2021.01.018.

5. Akdemir R, Gunduz H, Emiroglu Y, Uyan C. Myocardial bridging as a cause of acute myocardial infarction: a case report. BMC cardiovascular disorders. 2002 Sep 21;2:15. doi: 10.1186/1471-2261-2-15.

6. Erol N. Challenges in evaluation and management of children with myocardial bridging. Cardiology (Switzerland). 2021;34668(10):1-8. doi: $10.1159 / 000513900$.

7. Mohlenkamp S, Eggebrecht H, Ebralidze T, Monzberger S, Schweizer T, Quast B, et al. Muskelbrocken der Koronararterien: mogliche ischemierelevante Normvarianten [Normal coronary angiography with myocardial bridging: a variant possibly relevant for ischemia]. Herz. 2005 Feb 25;30(1):37-47. German. doi: 10.1007/s00059-005-2654-0.

8. Zeina AR, Odeh M, Blinder J, Rosenschein U, Barmeir E. Myocardial bridge: evaluation on MDCT. Am J Roentgenol. 2007;188(4):1069-73. doi: 10.2214/AJR.06.0714.

9. Almeida AR, Pereira AR, Morgado G, et al. Three-vessel myocardial bridging: a possible cause of myocardial stunning. Rev Port Cardiol. 2019;38(3):1-5. doi: 10.1016/j.repc.2017.09.030.

10. Ibarrola M. Myocardial bridges - a forgotten condition: a review. Clin Med Img Lib. 2021;7(1):1-8. doi: 10.23937/2474-3682/1510162

11. Alegria JR, Herrmann J, Holmes DR, Lerman A, Rihal CS. Myocardial bridging. Eur Heart J. 2005;26(12):1159-68. doi: 10.1093/eurheartj/ ehi203.

12. Hostiuc S, Negoi I, Rusu MC, Hostiuc M. Myocardial bridging: a meta-analysis of prevalence. J Forensic Sci. 2018;63(4):1176-85. doi: 10.1111/1556-4029.13665.

13. McCabe MJ, Weston CFM, Fraser AG. Acute myocardial infarction related to smoke inhalation and myocardial bridging. Postgrad Med J. 1992;68(803):758-61. doi: 10.1136/pgmj.68.803.758.

14. Hossain S, Uddin Ahmed M, Abdul Mannan M, Shahimur Parvez M, Shahimur Parvez D, Mahidur Rahman M, et al. Study of prevalence, risk factors and angiographic profile of patients with myocardial bridges in a Tertiary Care Hospital, Dhaka, Bangladesh. Cardiol Cardiovasc Res. 2020;4(2):67-70. doi: 10.11648/j.ccr.20200402.16

15. Slavich M, Patel RS. Coronary artery spasm: current knowledge and residualuncertainties. Int J Cardiol Heart Vasc. 2016;10:47-53. doi:10.1016/j.ijcha.2016.01.003.

16. World Health Organization. Tobacco responsible for $20 \%$ of deaths from coronary heart disease [Internet]. Geneva: WHO; 2018 [cited 2020 Dec 23]. Available from: https://www.who.int/news/item/22-09-2020tobacco-responsible-for-20-of-deaths-from-coronary-heart-disease

17. Bentley MC, Almstetter M, Arndt D, et al. Comprehensive chemical characterization of the aerosol generated by a heated tobacco product by untargeted screening. Anal Bioanal Chem. 2020;412(11):2675-2685. https://doi.org/10.1007/s00216-020-02502-1

18. Mallock N, Böss L, Burk R, et al. Levels of selected analytes in the emissions of "heat not burn" tobacco products that are relevant to assess human health risks. Arch Toxicol. 2018;92(6):2145-2149. doi.org/10.1007/ s00204-018-2215-y.

19. Polosa R, Rodu B, Caponnetto P, Maglia M, Raciti C. A fresh look at tobacco harm reduction: the case forthe electronic cigarette. Harm Reduct J. 2013;10:10-9. doi: 10.1186/1477-7517-10-19.

20. Biondi-Zoccai G, Sciarretta S, Bullen C, et al. Acute effects of heat-notburn, electronic vaping, and traditional tobacco combustion cigarettes: The Sapienza University of Rome - Vascular Assessment of Proatherosclerotic Effects of Smoking (SUR - VAPES) 2 Randomized Trial. J Am Heart Assoc. 2019;8(6):e010455. doi: 10.1161/JAHA.118.010455. 
21. U.S. Food and Drug Administration. Modified Risk Orders for tobacco products [Internet]. Washington: FDA; 2020 [cited 2020 Dec 23]. Available from: https://www.fda.gov/tobacco-products/advertising-andpromotion/modified-risk-orders

22. Galbraith EM, Eshtehardi P, Samady H. SPECT perfusion imaging and myocardial bridges: bridging the gap of diagnostic uncertainty. J Nucl Cardiol. 2011;18(6):1000-2. doi: 10.1007/s12350-011-9443-3.
23. Gómez FA, Forero PL, Ballesteros LE. Microscopic analysis of the myocardial bridges and their relationship with atheromatous plaque. Int J Morphol. 2021;39(1):70-6.

24. Apostolopoulou SC, Vagenakis GA, Sbarouni E. Multiple myocardial bridges causing severe ischaemia in adolescent with pulmonary stenosis. Cardiol Young. 2020;30(10):1530-1. doi: 10.1017/S1047951120002449.

\section{Authors' ORCID iDs and academic degrees}

Mihail Tasnic, MD, PhD Applicant - https://orcid.org/0000-0001-7004-4402

Ilia Catereniuc, MD, PhD, Professor - https://orcid.org/0000-0002-5479-4198

\section{Authors' contributions}

MT conceptualized the idea, conducted literature review, wrote the manuscript, revised and approved the final text; IC designed the research and revised the manuscript critically. Both authors revised and approved the final version of the manuscript.

\section{Funding}

This study was supported by Nicolae Testemitanu State University of Medicine and Pharmacy, The International Hospital Medpark and journal publication fee was covered by Philip Morris Sales and Marketing SRL. The authors are independent and take responsibility for the integrity of the data and accuracy of the data analysis.

\section{Ethics approval and consent to participate}

The project was approved by the Research Ethics Committee of Nicolae Testemitanu State University of Medicine and Pharmacy (protocol No 55 of 03.06.2016).

\section{Conflict of Interests}

No competing interests were disclosed. 